\title{
Sensor, Signal, and Imaging Informatics: Evidence-Based Health Informatics
}

\author{
S. Voros ${ }^{1}$, A. Moreau-Gaudry ${ }^{1,2}$, Section Editors for the IMIA Yearbook Section on Sensor, \\ Signal and Imaging Informatics \\ ' UJF-Grenoble 1 / CNRS / INSERM, TIMC-IMAG UMR 5525, Grenoble, F-38041, France \\ 2 UJF-Grenoble 1 / CHU / INSERM CIT803, Grenoble, F-38041, France
}

\begin{abstract}
Summary
Objectives: This synopsis presents a selection for the IMIA

(International Medical Informatics Association) Yearbook 2013

of excellent research in the broad field of Sensor, Signal, and

Imaging Informatics published in the year 2012.

Methods: We performed a systematic initial selection and a

double blind peer review process to find the best papers in

this domain published in 2012, from the PubMed and Web of

Science databases. A set of MeSH keywords provided by experts

was used.

Results: Current research in the field of sensor signal and imaging informatics is based on innovative sensors from which relevant information (signal and imaging) can be acquired and integrated into workflow based or patient based models. This

rich environment can help the medical staff in carrying out more optimal care. The demonstration of the added value of such innovations is more and more performed through evidence-based evaluations.

Conclusions: The best paper selection of articles on sensors, signal, and imaging informatics illustrates the wide spectrum covered by this field in 2012 , and the attention paid to evaluations.
\end{abstract}

\section{Keywords}

Evidence, biomedical informatics

Yearb Med Inform 2013:117-9

\section{Introduction}

The selection is composed of papers addressing innovative approaches in the field of sensor, signal, and imaging informatics naturally aiming at improving the patient care. The selection is organized according to three topics: perception, modelling and planning.

Perception addresses all means to acquire relevant information about the patient and his environment as exhaustively as possible. This information can then be used to create or instantiate models from which a planning is implemented in order to improve the medical care. All these innovative approaches are evaluated through different methods, according to their degree of maturity.

Since the proposed systems (hardware or software) are considered "Medical Devices" from a regulatory point of view, evidence-based evaluation is becoming more important in order to demonstrate their added-value and contribution to the benefit/risk ratio.

Our selection process was based on the extraction of Sensors, Signal, and Imaging Informatics articles in PubMed and Web of Science using an exhaustive list of MeSH headings and major topics. This search returned 7,200 articles on PubMed only. We retained 2,000 PubMed articles satisfying the MeSH query: The papers come from 17 of the most representative journals in terms of number of articles that matched the MeSH query. We finally added 5 non-represented journals that we considered major in this field. We thus studied 2,399 articles from PubMed and 812 articles from Web of Science (after duplicates removal).

\section{Perception}

In order to make the "best" therapeutic decisions for a patient, there is a need for devices to allow:
- To see inside tissues (for instance to localize a tumor). To do so, devices [1] may use shear-wave elastography, or [2] a photoacoustic and ultrasound imaging system based on the Laser-Ultrasonics optical technique.

- To characterize the quality of a tissue. The malignancy of a tissue can be determined without contact thanks to hyperspectral imaging [3] or spectroscopic imaging [4]. On the other hand, [5] presents a contact-based approach for discriminating between three tissue types (muscle, fat and blood) with the development of a broadband impedance spectroscopy needle.

\section{Modelling}

Once the relevant data is acquired, it can be integrated into a model, which allows for numerical structuring of the knowledge domain. As a result of the modelling it is then possible to draw conclusions to perform the right therapeutic action.

Models can be built to describe surgical procedures. [6] uses such a model to predict surgery times and support the scheduling of the operation theatre. [7] automatically identifies the current surgical phase or action with the goal of triggering events or assistance that are required or desired at specific moments during surgery.

Models can also be constructed to represent complex anatomical structures. For instance, [8] developed an active cardiac model for the cardiac structure detection of fetal images during ultrasound, and [9] uses an a priori model of a rectal ultrasound probe in order to track the deformations and displacements of the prostate gland during its examination in real-time. 


\section{Planning}

Ultimately, data acquisition and models can be exploited to help the practitioner carry out a medical procedure optimally: in [8], the automatic cardiac structure detection is a support tool for the early diagnosis of congenital heart defects. In [9], the determination of the prostate's displacement allows for a better control of prostate biopsies. Finally, [10] proposes a planning software for neurosurgery where the user can identify the surgical instrument's trajectory that will minimize the risk for the patient.

\section{Evaluation}

All the above papers present medical devices at different levels of maturity. This variation explains why different kinds of evaluations have been performed to estimate or demonstrate the benefit of these medical devices.

Some evaluations consist of pre-clinical experiments on a small amount of tissue (e.g. $[2,4,5])$ or clinical data (e.g. $[7,8]$ ) and experiments on small animals (e.g. [3]).

The next level of evaluation consists generally in a small-scale single center clinical evaluation where the innovation is exposed to the clinical reality. This provides first estimates if the medical service is delivered effectively by the medical device (e.g. $[6,9,10]$ ).

These first evaluations are then followed and extended by clinical evaluations on larger cohorts of patients to expose the device to a large-scale use. For instance in [11], 182 women were involved in the evaluation of a biofield diagnostic system for breast cancer detection. In [1] 958 women took part in a multinational study on shear-wave elastography for improving the specificity of breast cancer detection.

\section{Conclusion}

The best paper selection for the Yearbook section 'Sensor, Signal and Imaging Informatics' resulted in the identification of several thousand of articles; reflecting the breadth of this extremely active field. A first double blind selection on abstracts led to 37 articles. These papers were fully reviewed and graded, leading to the selection of papers described here.

The five best papers selected by the peer-reviewers reflect the abundance of

Table 1 Best paper selection of articles for the IMIA Yearbook of Medical Informatics 2013 in the sections 'Sensor, Sginal and Imaging Informatics'. The articles are listed in alphabetical order of the first author's surname.

\section{Section}

Sensor Signal and Imaging Informatics

- Baumann M, Mozer P, Daanen V, Troccaz J. Prostate biopsy tracking with deformation estimation. Med Image Anal 2012 Apr; $16(3): 562-76$.

- Deng Y, Wang Y, Shen Y, Chen P. Active cardiac model and its application on structure detection from early fetal ultrasound sequences. Comput Med Imaging Graph 2012 Apr;36(3):239-47.

- Padoy N, Blum T, Ahmadi SA, Feussner H, Berger MO, Navab N. Statistical modeling and recognition of surgical workflow. Med Image Anal 2012 Apr; 16(3):632-41.

- Shamir RR, Joskowicz L, Tamir I, Dabool E, Pertman L, Ben-Ami A, Shoshan Y. Reduced risk trajectory planning in image-guided keyhole neurosurgery. Med Phys 2012 May;39(5):2885-95.

- Trebbels D, Fellhauer F, Jugl M, Haimerl G, Min M, Zengerle R. Online tissue discrimination for transcutaneous needle guidance applications using broadband impedance spectroscopy. IEEE Trans Biomed Eng 2012 Feb;59(2):494-503.

the current research trends in the field. As illustrated by these articles, more and more importance is attached to Evidence-Based health informatics.

\section{Acknowledgments}

We acknowledge the contributions of the Rouen CHU CISMeF researchers for their constitution of the MesH query, Martina Hutter, and the reviewers in the selection process of the IMIA Yearbook.

\section{References}

1. Berg WA, Cosgrove DO, Doré CJ, Schäfer FK, Svensson WE, Hooley RJ, et al.; BE1 Investigators. Shear-wave elastography improves the specificity of breast US: the BE1 multinational study of 939 masses. Radiology 2012 Feb;262(2):435-49.

2. Rousseau G, Gauthier B, Blouin A, Monchalin JP., Non-contact biomedical photoacoustic and ultrasound imaging. J Biomed Opt 2012 Jun;17(6):061217.

3. Akbari H, Halig LV, Schuster DM, Osunkoya A, Master V, Nieh PT, et al. Hyperspectral imaging and quantitative analysis for prostate cancer detection. J Biomed Opt 2012 Jul;17(7):076005

4. Lue N, Kang JW, Yu CC, Barman I, Dingari NC, Feld MS. Portable optical fiber probe-based spectroscopic scanner for rapid cancer diagnosis: a new tool for intraoperative margin assessment. PLoS One 2012;7(1):e30887.

5. Trebbels D, Fellhauer F, Jugl M, Haimerl G, Min $\mathrm{M}$, Zengerle R. Online tissue discrimination for transcutaneous needle guidance applications using broadband impedance spectroscopy. IEEE Trans Biomed Eng 2012 Feb;59(2):494-503.

6. Devi SP, Rao KS, Sangeetha SS. Prediction of surgery times and scheduling of operation theaters in ophthalmology department. J Med Syst 2012 Apr;36(2):415-30.

7. Padoy N, Blum T, Ahmadi SA, Feussner H, Berger MO, Navab N. Statistical modeling and recognition of surgical workflow. Med Image Anal 2012 Apr;
16(3):632-41

8. Deng Y, Wang Y, Shen Y, Chen P. Active cardiac model and its application on structure detection from early fetal ultrasound sequences. Comput Med Imaging Graph 2012 Apr; 36(3):239-47.

9. Baumann M, Mozer P, Daanen V, Troccaz J. Prostate biopsy tracking with deformation estimation. Med Image Anal 2012 Apr; 16(3):562-76.

10. Shamir RR, Joskowicz L, Tamir I, Dabool E, Pertman L, Ben-Ami A. Reduced risk trajectory planning in image-guided keyhole neurosurgery. Med Phys 2012 May; 39(5):2885-95.

11. Subbhuraam VS, Ng EY, Kaw G, Acharya U R, Chong BK. Evaluation of the efficiency of biofield diagnostic system in breast cancer detection using clinical study results and classifiers. J Med Syst 2012 Feb;36(1):15-24.

\section{Correspondence to:}

Sandrine Voros

Laboratoire TIMC-IMAG, équipe GMCAO

IN3S, pavillon Taillefer

Faculté de Médecine

38706 La Tronche Cedex, France

Tel: + 33456520009

Fax +33456520055

E-mail:Sandrine.Voros@imag.fr

\section{Appendix: Content Sum-} maries of the Selected Best Papers for the IMIA Yearbook 2013, Section Sensors, Signal and Imaging Informatics ${ }^{1}$

\footnotetext{
The complete papers can be accessed in the Yearbook's full electronic version, provided that the article is freely accesible or that your institution has access to the respective journal.
} 
Padoy N, Blum T, Ahmadi SA, Feussner H, Berger MO, Navab N

Statistical modeling and recognition of surgical workflow

Med Image Anal 2012 Apr; 16(3):632-41

The workflow inside the Operating Room (OR) has an impact on the working conditions of the surgical staff and the hospital operations in general. In order to assist the surgical staff to perform various tasks (e.g. automation of simple tasks, failure detection, documenting procedures), Padoy et al. propose two statistical models constructed from generic OR signals for delayed or real-time recognition of the surgical phases in a standard endoscopic surgery: $n$ average surgery model using the Dynamic Time Warping algorithm, and Hidden Markov Models (HMM). The approach was validated using 16 laparoscopic cholecystectomies performed by four surgeons of varying expertise, with the surgical instruments use as model input. A video labeling software allowed the author to manually indicate which surgical instruments were present at each time-step with a temporal resolution of one second. The authors obtained a phase recognition accuracy ranging between 88-97\% for off-line detection and ranging between $84-89 \%$ for on-line recognition, depending on the selected model.

\section{Baumann M, Mozer P, Daanen V, Troccaz J Prostate biopsy tracking with deformation estimation}

\section{Med Image Anal 2012 Apr;16(3):562-76}

Today, prostate biopsies are the only definitive way to confirm a prostate cancer. These biopsies are performed according to a standard procedure that suffers from several shortcomings. The authors propose a 3D transrectal ultrasound (TRUS) based tracking system for prostate motion occurring during transrectal biopsy acquisition. This approach is purely image-registration based, and enables the user to identify the position of the probe with respect to the gland by using a kinematic model of the rectal probe motion. Armed with this knowledge, the authors were able to compute precise biopsy and cancer maps, to project the TRUS target into Magnetic Resonance Imaging (MRI) volumes, to create MRI Biopsy maps to plan therapy, and provide rudimentary support to guide towards non-ultrasound targets. This innovative approach was then evaluated through clinical research, with a Root Mean Square (RMS) error of $3.6 \mathrm{~mm}$ by the system.

\section{Shamir RR, Joskowicz L, Tamir I, Dabool E, Pertman L, Ben-Ami A, Shoshan Y \\ Reduced risk trajectory planning in im- age-guided keyhole neurosurgery Med Phys 2012 May;39(5):2885-95}

Preoperative planning of trajectories for straight rigid tool insertion in image-guided keyhole neurosurgery is crucial in order to prevent peri-operative complications. The authors developed and evaluated a new computer assisted approach to choose the "best" trajectory, i.e. the trajectory that most reduces the risk of the planned surgery. The risk quantification is computed by taking into account the surgeon defined target and a multi-parameter risk card based on proximity of the planed trajectory to critical brain structures. This approach is supported by allowing the surgeon to adapt his future trajectory with the help of an interactive $3 \mathrm{D}$ visualization of surrounding structures. The enhanced environment to plan trajectories was validated with a comparative retrospective review of MRI head scans for eight patients. The results show a significant reduction in insertion trajectory risk with a reduction of time gain required to plan the procedure and an improvement in the understanding of possible risks and spatial relations of the trajectory and patient anatomy.

\section{Trebbels D, Fellhauer F, Jugl M, Haimerl G, Min M, Zengerle R \\ Online tissue discrimination for transcuta- neous needle guidance applications using broadband impedance spectroscopy}

\section{IEEE Trans Biomed Eng 2012 Feb;59(2):494-503}

During clinical procedures involving needles, it is essential to be able to check that the needle tip is effectively inside the pre-defined tissue target. Although different imaging approaches exist, Trebbels $\mathrm{D}$ and al. propose an innovative approach, which does not involve imaging techniques. The authors developed and simulated a new medical device including a hollow coaxial needle that enables the real-time measurement of the impedance spectra of a biological tissue close to its tip. This measure is achieved by acquiring broadband chirp signals at the needle tip from which the transfer function of the tissue in the frequency domain is computed. After having demonstrated in silico the feasibility of the approach, ex vivo and in vivo experiments on anesthetized pigs were conducted to characterize the tissue type at the needle tip. Such tissue classification is obtained by establishing the correlation of the measured complex transfer functions with known library functions. The obtained results showed two distinct distributions of correlation coefficient when tissue types match the library functions or not. Using the distributions, the authors were able to identify three tested tissues types (muscle, fat, and blood) accurately.

\section{Deng Y, Wang Y, Shen Y, Chen P \\ Active cardiac model and its application on structure detection from early fetal ultra- sound sequences}

\section{Comput Med Imaging Graph 2012 Apr;36(3):239-47}

Aiming to assist the diagnosis of fetal heart abnormalities, Deng et al. propose a new method for the early detection of fetal heart structure on Ultrasound sequences. The approach is based on a pre-processing phase and an active cardiac model which considers both structure and motion information. In the preprocessing phase, a Region of Interest (ROI) is automatically determined by identifying the high "energy" region from accumulated motion images over the ultrasound sequence, and a despeckling process is applied to the ROI in order to reduce the image noise. The fetal heart is then modeled with eight spatially linked parts, whose locations can evolve over the ultrasound sequence. The active cardiac model parameters are learned based on a manual segmentation of a training sequence. Experimental validation on six testing sequences demonstrated that the proposed approach outperformed state-ofthe-art methods with an error less than 10\% of the ROI width for $90 \%$ of the detections. 